\title{
Modeling Ontogeny in Biology
}

\author{
Yury N. Zhuravlev, Mikhail A. Guzev and Evgeny E. Skurikhin
}

\begin{abstract}
Certain general events in ontogeny and genetic information expression in biological objects are defined to aid in their description in terms of algebraic topology, geometry and category theory. We begin by defining chromatin as an information matrix that generates a number of bases for the following mapping, and we consider these events as fibering the first range on the chromatin. Each such base of the first range can be further decomposed into germs, which are represented by transcripts of different classes of equivalence, such as mRNAs, rRNAs, tRNAs and many others. Considering the germs as elements of biological object as a system, we follow their transmutation trajectories to the moment that they cross into the translation process. This crossing is interpreted here as a central point where the global genetic code information, local mRNA information, and global chemical information combine to facilitate the construction of molecular, supramolecular, and more complex biological 'instruments' that interact with their surroundings. The notion of gluing is used to describe the processes of crossing and merging the trajectories of the primary phenotypic elements that construct the bundles of phenotypic elements and the phenotype.
\end{abstract}

Keywords-genotype-phenotype mapping, transcription, choice, base, translation, global and local information

\section{Introduction}

The nature of biological objects (singularity of their construction and behavior) yields specific modelling requirements for biology.

First, such a complex entity as a biological object (BO) cannot be simplistically defined. BOs are characterized by individual development, lifespan and exclusively complex relationships with their surroundings.

Different groups of authors have selected different classes of BO traits based on their capacity to represent life and living systems (e.g. [1,2]). Seemingly, defining the singularity(ies) for life is a difficult (if solvable) problem for which certain questions remain unsolved and occasionally ignored in scientific discussions [3].

Another difficulty is the problem with selecting an archetype or general image of a living system for modelling. A widespread conviction is that "life is composed of organisms", which often leads scholars to use "cell" or "organism" as

Yury N. Zhuravlev

Institute of Biology and Soil Science FEB RAS

Russian Federation

Mikhail A. Guzev, Evgeny E. Skurikhin

Institute for Applied Mathematics FEB RAS

Russian Federation synonyms for life (see discussion in [4]). Accordingly, a model of the cell is considered a model of a biological object (BO) in most modern publications. However, a locally selected cell represents a BO in a reduced and depleted state; it primarily concerns characteristics of living systems, such as movement (becoming and creation), complexity and inseparability, the carries for which are unknown. A recent attempt to model the living cell at the molecular level using formal language demonstrates how many new properties of living systems can be discovered using this technique [5].

To reflect these singularities in life systems, the notion that BO must have two general definitions was developed [6,7]. Authors believe that a BO cannot be exhaustively defined by only using genetic or phenotypic constituents. The internal and external BO definitions must be combined. The internal definition is mainly genetic and considers the $\mathrm{BO}$ a triad that includes $O=(P, F, P h)$, where the sequences $(p)$, mapping $(f)$, and phenotypic $(p h)$ participants compose the object. The external definition reflects the BO's position and role in its surroundings (living and non-living in unity). Here, the object can be interpreted as a certain operator that converts the surroundings: $O_{i n}=\Phi: \varphi\left(O_{1}\right) \rightarrow O_{2}, O_{i n} \neq O_{1}, O_{2}, \varphi \in \Phi$. This definition is more ecological. No single definition can alone sufficiently describe the BO. However, even both definitions combined are insufficient to define life as a system because the multitude of BOs only comprise a list of elements related to life, whereas producing the system from its elements produces a novelty that cannot be directly constructed based on the elements' characteristics. Thus, defining life as a system must include characteristics absent in the BO definitions. The potential infinity of life (in contrast to the finite nature of objects) is an example of such novel characteristics.

Generally, individual development can be understood as a means to represent $\mathrm{BO}$ as the same object internally and externally [7]. Alternatively and more suitable for categorical representation, individual development is an object's path from birth to its final functional representation in the surroundings. In fact, in analyzing the individual development of an object, we consider its two fundamentally different presentations; this requires at least two fundamentally different descriptions: one using structural dynamics and the other using functional dynamics. The readiness of modern biologists to operate using these two types of descriptions differs; and the second type of description is often addressed to the ecological scope. Because we will focus on the internal definition related to individual development, we mainly remain in the biological scope.

We present our work in the following way: after a short and compressed description of molecular events significant for individual development in biological systems, we schematically represent the positions of these events in the space of ontogeny and, finally, speculate on which algebraic 
topology or geometric mechanisms can be used to model the transmutations of these symbolic entities to provide the material and informational content in living bodies.

\section{Background}

The notions of modelling and reduction are tied inseparably, but it is of importance which features of object modelled can be discarded and which must be conserved in model. That especially relates to the modelling living systems possessing unique organization, where transmutations of material carriers and their informational contents are concatenated.

In our attempt to select certain BO characteristics necessary for modelling, we began with seminal works by C.H. Waddington [8], R. Rosen [9], and H. Pattee [10]. Separately, the positions of each author can include controversial, unclear and even challenged points, but taken together, their works yield two important generalizations. First, they indicate that BOs are comprised of two distinguishable moieties, genotype and phenotype and are, therefore, composite objects. The BO structure is hierarchical and complex, so allocation of compositional attribute of $\mathrm{BO}$ is significant for description of its construction. Second, genotype and phenotype maintain a causal relationship that is typically represented as a map of $G \rightarrow P h$. From the ontogeny viewpoint, the events that 'fill' the arrow are important and direct our attention to category theory. Typically, category theory is the final point in a formal description of genotypephenotype mapping due to the general character of this theory. This difficulty can be overcome through reorganizing molecular biology data. We suppose that the processes that fill the arrow (which are, therefore, morphisms) are especially informative for BO modeling. Rating and assessing molecular events that connect the genotypic preimage of a $\mathrm{BO}$ with its ecological representative (phenotype) aid in differentiating these events into many classes of equivalence, and different mathematical models are necessary to describe the natural transformations of the classes as categorical objects. However, these natural transformations are far from linear and independent; they form a net with an intricate configuration and become interconnected and interdependent. Further, they can be elements that represent the biological world on a global or local scale or an intermediate position.

We believe that the first step must be geometrization of molecular knowledge that will facilitate the use of molecular data as bases for topological and geometric-algebraic models.

In fact, the aim of this publication is to differentiate and classify the processes involved in forming a BO. Therefore, we concentrate solely on the ontogeny-related processes. However, such differentiation and classification is impossible due to the general positions that are somewhat pompously referred to as biological axioms. This article considers one such axiom: the axiom of conditionality or incompleteness for local representation. This axiom indicates that any representation of a biological object and the allocation of its internal and external relations, among other concerns, is incomplete and conditional. The axiom is inconsistent with the practice of using autonomy as criteria for life (see reviews in [11]. However, new results in a study on the vertebrata biome [12] create doubt in the validity of this criterion. As an alternative, the notion of a multi- or metagenomic state is considered, wherein the metagenomic state is assumed to be the usual state of an organismal organization [13]. The effects of conditionality can also extend into the description of individual development. Thus, the caterpillar can be considered a stationary organizational state of an insect; during that development stage, it is always a caterpillar. However, at almost every moment of this stage, the caterpillar is growing; its cells divide and tissues differentiate. It is always in construction; thus, the notion that her state does not depend on time is conditional and incomplete.

One of the most expressive examples of conditionality is the recent discovery that maternal plasma DNA is a mixture of maternal and fetal DNA [14]. Combined with known events in exchange between DNA fragments and even organelles, such as between scion and rootstock [15], these phenomena demonstrate widespread conditionality in nature.

Therefore, although herein we only study (conditionally) the path from genotype to phenotype, other entities and notions are involved in our models.

\section{Ontogeny Definitions}

\section{A. Objects or Operational Units}

To represent ontogeny in terms of category theory, we should be more attentive to mappings as morphisms; however, topological specificity of the biological world demands a careful description and interpretation of the operational units that compose the BO. For $G \rightarrow P h$ mapping, an important unit is chromatin, but interim genetic bases, operational organelles, and organs are also significant.

\section{1) Chromatin as an IM}

Chromatin was discovered based on its specific staining and is still often defined by this property. In the Encyclopedia Britannica, the following is presented: «This combination of DNA with proteins creates a dense, compact fibre called chromatin» [16]. This is true, but paradoxically, numerous experiments show that chromatin performs its functions more often when it is invisible and not when it is colored and visible. Chromatin is much more important for interpretation as a basic information structure for an object (cell, organ, organism and even a population). When the net presentation is consistent with organization of the concerned biological units, chromatin forms a network, which is the basis for most other network manifestations of cellular and organismal dynamics. Chromatin is characterized by diverse dualism, continuous dynamics and a number of other properties that support its characterization as an information matrix. The concept of chromatin as an information matrix (IM) is based on the statement that any heterogeneous structure for which the heterogeneity is stable can be considered an IM under the stipulation that at each regular consideration, the nature and details of such heterogeneity are (almost) the same as the previous consideration. The conventionality of this definition 
reflects the conventionality of the object(s) we consider in biology. IMs can carry diverse (potential) information, which can equal zero and infinity depending on the availability of specific decoders and the scale of consideration.

In introducing the concept of IM, we introduce a new type of evolutionary dualism, wherein we set a class of matrices against a class of decoders. In a sense, introducing the matrices/decoders dualism approximates a $\mathrm{BO}$ to quantum entities. Lewis [17:715] believes that in representing quantum mechanics, the configuration space has information that is absent in the individual wavefunction. Similarly, the biological world does not contain information that fully belongs to chromatin or nucleic acids; the biological world includes such information only as far as the cytoplasm, adjacent cell or even another (outer) cell or organism have decoders. Without the ribosome, the triplet sequences do not carry any such information and the female insect's pheromones only provide information to males of the same species.

An IM's specificity consists in that it operates in a specific variant/invariant regime such that after legal transformations, it returns to a state that is similar to the initial state, thus demonstrating the principal and distinctive feature of life, in which the quantity of stored information largely exceeds the system fluctuations [18]. Thus, an IM behaves as a 'propagating organization' as defined by Kauffman et al. [19].

\section{2) Classes of Transcripts as Interim Bases for Mappings}

Chromatin activity results in producing transcripts with a broad spectrum of potential. The most studied groups of transcripts include mRNAs, tRNAs, rRNAs and regulatory transcripts, such as short and long RNAs.

Considering these classes as interim bases for the corresponding maps, due to initial mapping, the single unit chromatin base yields the number of smaller bases for transcripts. These bases are objects for different morphisms; rRNAs are used to structure the ribosome backbone, tRNAs aid in carrying activated amino acid residues, and mRNAs deliver information for protein synthesis [20].

For topology, these events can be interpreted as fiber bundles that decompose a covalently bound supramolecular structure into the final number of sets of free transcripts. In certain cases, decomposition can continue to generate elementary structures (germs), whereas in other cases, certain interim structures (germ and bundle combinations) can be constructed.

\section{3) Germs as Base Elements}

The aforementioned small bases are constructed by elements that, operationally, belong to the same class of equivalency but can differ structurally. Thus, all mRNA are matrices for protein synthesis, whereas a unique sequence of monomer nucleotides is characteristic for most mRNAs. Similarly, tRNAs are subdivided in subclasses that are specific for the assigned amino acids, and inside such subclasses, they differ structurally, which is reflected in the synthesis mechanism for such basic elements. Thus, in eukaryotes, rRNA base construction begins through synthesizing a $5 \mathrm{~S}$

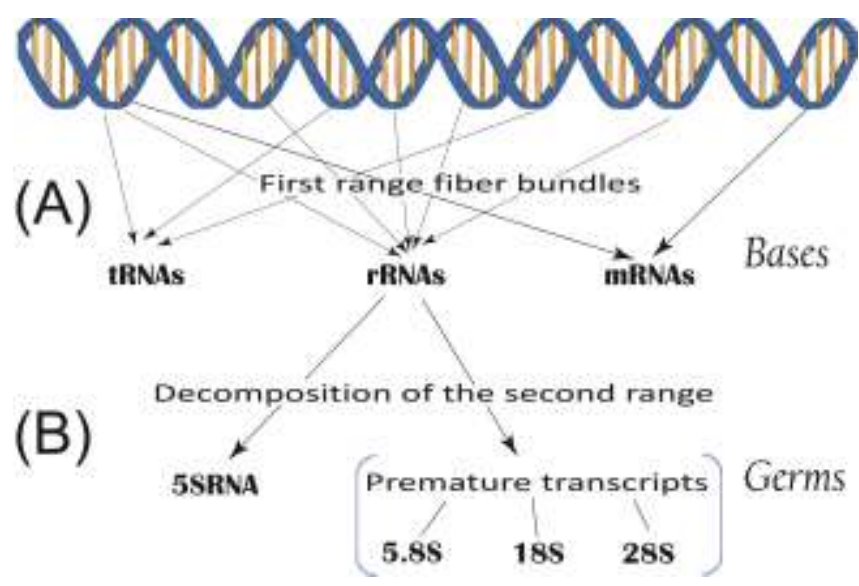

Figure 1. Choice generated bases as two steps of inverse projection.

A - fibering the first range generated on an IM: local informational transcripts, such as mRNAs; global (on the genetic code scale) informational transcripts, such as tRNAs; structure constructing transcripts, such as rRNAs; and regulatory transcripts with known and unknown functions, such as short and long RNAs.

$\mathrm{B}$ - fibering the second range as the decomposition of rRNA bases into germs represented by individual rRNA molecules.

rRNA and pre-mature rRNA transcript. Maturation of the latter transcript includes processing, after which the primordial transcript (13000 nucleotides long) is deconstructed into 28S, $18 \mathrm{~S}$, and 5.8S fragments. Fragments undergo nucleotide Nbase modification, intron splicing, and 3'- as well as 5'-end modification. These and other transformations can be considered conversion of tRNAs basic elements, whereas their inclusion in the backbone structure of a future ribosome is the first step in gluing (Fig. 1; see also the next section).

The aforementioned transcripts are polymeric molecules with complicated structure; however, in the context of fiber bundles for developing BOs, we must accept these molecules as elementary, indivisible base elements for the corresponding range. Moreover, in systems with a significant number of interim bases, basic elements can comprise even more complex structures. Nevertheless, this structural complexity admits their representation as minimal functional (operational) elements of base.

\section{B. Morphisms}

Here, we introduce certain definitions that classify the mapping processes, which are symbolized by an arrow in the general formula $G \rightarrow P h$.

Choice - Mapping for a set, which defines a certain subset of the set. The singularity of the BO sets permits a set of choices and subset selections from either a set of chemically distinct elements or set-unity (e.g., a covalently bound supramolecule).

Transformation - A class of maps that convert one structure into another and conserve certain fragments of the first structure. Such map classes include splicing events that convert pre-mRNA into mRNA. In more complex cases, 
differentiation or dedifferentiation of cells and tissues can be interpreted as transformations.

Construction - A class of maps that construct a new structure based on information contained in another structure. In this case, fragments of the initial structure are not conserved in the constructed structure.

Map, mapping - Genotype-phenotype mapping that is represented as mapping $\varphi$ from the elements of a genospace, which is denoted as $G$, into elements of certain phenospace, which is denoted as $P$ (after [21]; see also [22]). However, in this paper, we use the term in its broad sense; thus, the aforementioned transmutations of objects and operational units can be considered mappings.

Gluing - An operation with germs and germ bundles that produces the structures with specific qualities for phenotypic traits. In topology, the gluing process generates new properties for a system. This may be a method for generating novelties in BO ontogeny.

Molecular events in interim genetic base production are shown in Fig. 1. The selective choices on chromatin matrix map the selected segment of DNA into distributed set of bases of transcripts some of which are presented in schema (tRNAs, rRNAs, and mRNAs). Many other bases, such as repetitive sequences, transposons, and epigenetic regulation elements, are omitted here. However, many of these bases can be fibrated further to produce elementary information carriers, such as germs.

For consistency between the intuitive notion of fibering genetic material in the cell and the common schematics for fiber bundles in topology, the projection direction must be considered. Then fiber bundle is a surjective mapping of the space $F$ onto the space $I, p: F \rightarrow I$, where the space $I$ is referred to as the base space of the bundle, and $F$ is the total space for the bundle (see also [23]); the map $p$ is referred to as the projection map (or bundle projection) and defined the inverse image, $p^{-1}$, which can be considered a multivalued map or a set-valued function of the space $I$. The inverse image of a set member, $p^{-1}(i)$, is also referred to as the fiber over $i$, or the stalk. Considering biological (and sociological) analogies, members of $F_{\mathrm{i}}=p^{-1}(i)$ will often be referred to as the germs at $i$. Notably, members of the stalk of sheaf are also referred to as the germs.

Because $p$ is a general (not multivalued) map, the fibers are pairwise disjointed (e.g., $F_{\mathrm{i}} \cap F_{\mathrm{j}}=\varnothing$ ). We do not suppose that $F$ and $I$ are topological spaces, but as the need arises, we fix on them the structures considerable in the range of questions at issue.

\section{Three Steps of Ontogeny}

The central dogma of molecular biology [24] prescribes that we consider ontogeny as the map $G \rightarrow P h$, where the succession of directed information transfer DNA $\rightarrow$ RNA $\rightarrow$ Protein is generally valid. Currently, most biologists agree that this concept is simplistic, flat, and incomplete [25]. The following question arises: what transformations can convert this generally valid concept into a complex, eidetic, and complete notion? We believe that the corresponding geometrization and formalization of molecular data can be fruitful, although correspondence between genotype and phenotype organization is generally tacit.

\section{Step 1, decomposition.}

In classical representations, for multicellular organisms (e.g., in insects), the BO life begins through gamete union and continues as a succession of development stages, including multiple molts, maggot, nymph, caterpillar, pupa, and adult (imago). The phenomenon of death, which generally occurs after (or as a result of) reproduction, completes the process.

In molecular representations, constructing a future insect organism begins much earlier or, at least, simultaneously with the beginning of gametogenesis. This process can be interpreted as a type of fibering; however, fibering on the chromatin IM is clearer. These events begin with selecting a subset of activated segments in chromatin. Transcription factors and short RNAs of a parental nature are effectors in this choice [26, 27].

The gene coding portions of the selected segments are transcribed to construct the interim base of transcripts (Fig. 1. For details on the splicing mechanism, see [28]). These bases are constructed de novo because material portions of chromatin segments are not converted into transcript bodies; only the 'naked' information is transferred into transcripts. In contrast, material bodies of pre-mRNA transcripts are converted into mature mRNA bodies for translation onto ribosomes.

Step 2, conversion cannot be distinctly represented because each germ and trajectory undergoes conversion in a specific place and time. Thus, translation products appear due to de novo construction using the mRNA information that the material body discards (see also the next section). However, the transmutation of participants in this process can be interpreted as a conversion. The conversion step explicitly demonstrates that certain distributed discrete processes in ontogeny do not contain a phase transition with clear common border.

\section{Step 3, gluing}

BO construction is completed through processes that glue converted germs and germ bundles into a conditionally discrete and united entity. At this development stage, the most portion of the object is represented by structures related to the phenotypic traits (observables). The genetic moiety of a BO is protected from external effects through quality-bearing phenotype properties. Nevertheless, genotypic and phenotypic structures coexist in the $\mathrm{BO}$ and convert its body into a composite entity. The manner used to describe gluing the germs and germ bundles as well as the union of genetic and phenetic moieties is decisive for the ontological model of complexity. 


\section{A. Informational Content in Ontogeny Mapping}

According to common schematics for the information deployment in the cell [29], mature mRNA leaves the nucleus and enters the cytoplasm, where it meets ribosome and a set of tRNAs. The complete set of tRNAs is composed of dozens of molecules because, among the tRNAs that specifically accept an assigned amino acid, many (up to 6) are isoaccepting. Their trajectories begin from the IM, where the interim tRNA bases are constructed. The trajectory step includes aminoacyl tRNA synthases, which are enzymes that specifically load a tRNA with the corresponding amino acid [30]. Notably, these enzymes originate from mRNA interim bases. Enzymes transform tRNAs into aminoacyl tRNAs, and the latter tRNAs are principal components for protein synthesis. These components are not involved in chemical connections or direct order relationships before they meet at the ribosome [31].

However, the informational events that precede this meeting are interesting.

Above, we considered that the molecular body and information portion trajectories can diverge. Only local portions of information are explicitly joined to such molecules as mRNA and rRNA. The information on the genetic code rules is distributed among all of the biological world participants and must be considered global for the entire biological world. Moreover, much information on the rules of interactions in the organic world is involved in such complex processes as translation. In Fig 2, this information is represented as local genetic, G-Local; global genetic, GGlobal; and non-genetic, non-G. These types of information co-operate in converting the local information for symbol sequences (triplets are symbolic units for mRNA) into a sequence of protein shape elements (alpha helices, beta sheets, various pins and more unique substructures that are shaped elements). Later, in folding process, the sequence of shaped elements will be transformed into a globe-like shape of tertiary structure.

The events following the conversion of sequence information into form information are principally significant in phenotype generation. We can say these types of information cooperate because we do not understand exactly the nature of such information. However, we cannot say that these shaped element sequences glue to construct the phenotype because we know the material nature of these molecules. Therefore, speculation on gluing must be compatible with the general (global) notions underlying molecular interactions. This concept is one of the most intriguing points in ontogeny and should be considered in a separate publication. Here, we note that gluing involves not only germs and germ bundles but also other information with global meaning. The two arrows without content in Fig. 2 represent this hierarchical characteristic of gluing, the nature of which must be investigated and formalized.

This formalization promises to be an uneasy task because a number of hierarchies construct the complex and composite BO body. Practically, each interim base produces its own class of germs with a specific mode of gluing. Moreover, different

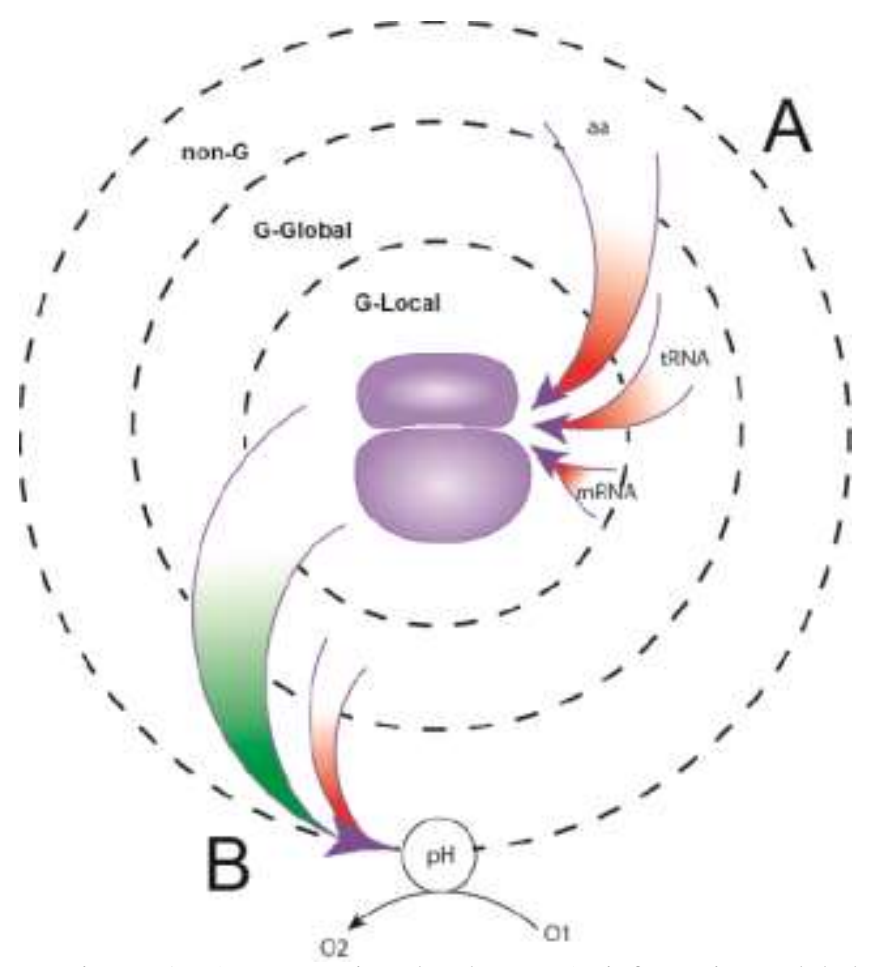

Figure 2: A - Merging local mRNA information, global genetic code information, and non-genetic information for amino acids to produce shape-specific protein information. The arrows indicate information pathways; certain material carriers are discarded.

B - Gluing shape-specific germs and germ bundles to create shape-bearing phenotype properties. The arrows indicate the material entities' pathways for which gluing produces the material construction, carriers of phenotypic properties.

gluing variants are possible between germs from different bundles as well as gluing between bundles and between bundles of bundles.

Different mathematical constructions, such as direct sum as well as direct and tensor products, are expected and should be used in this formalization.

\section{v. Global Categories That Are Important for Ontogeny}

Even where it is presented through numerous details, the formula $G \rightarrow P h$ cannot exhaustively represent ontogeny. The matter of incomplete representation of ontogeny with the formula is not necessary due to two BO definitions. An additional set of entities' trajectories are often not considered by embryologists because these germs are continuous among generations. Such entities include the cell membrane, cytoskeleton, cell wall in plants, and other structures that pass, in division, from the parental cell to the daughter cells via processes that are not clearly connected with chromatin activity [32]. Thus, their trajectories do not begin with the IM (understood as chromatin), but they are passed from cell to cell as if they exist continuously. Such behavior can indicate that the set elements begin at the beginning of the object 
origin, even before the $\mathrm{BO}$ origin (as a part of parental prebiological object(s)). Because they are not directly based on genotype, these set elements are perceived as phenotypic traits because they participate in interactions between the BO and surroundings. For example, the outer membrane, plasmalemma, controls water and inorganic element transport. However, plasmalemma can carry their own informational content from generation to generation, thus demonstrating a type of inheritance.

This distribution pattern for biological information in the living world has been noted by other authors: "Bioinformation resides in digital sequences in molecules such as DNA and RNA, three-dimensional structures, chemical modifications, chemical activities, both of small molecules and enzymes, and in other components and properties of biological systems..." [33].

This type of information which is associated with carriers differing from elements of Central dogma of molecular biology [24] is naturally to classify as global one. The significance of this information in the reconstruction of prebiological stages of evolution becomes evident.

\section{vi. Conclusion}

We began this work with the statement that proper geometrization of molecular data is necessary for developing a corresponding ontogeny model. After first rather timidly applying the category theory and general topology to describe BO dynamics, we found that ontogeny is represented by two types of flow: information and material; these types of flow can merge and diverge to generate specific, characteristic hierarchy patterns.

Many interesting aspects, which are not considered in the common ontogeny model, can be considered by carefully examining the organization and implementation mechanisms for these two types of flow. We observed that choice generates the interim bases for genotype $\rightarrow$ phenotype mapping and only occurs in systems with a consolidated IM. The notion of interim base introduced in our paper is important in modeling the biological complexity. The element of such bases is the simplest one in the operational sense; it cannot be reduced further without loss of its function; however it remains complex in the sense of its structural organization because it is represented by a specific construction. Direct sum and tensor product are supposed to be the mathematical representations of such constructions.

Fibering, conversion, and gluing are repetitive operations on a different scale of BO development. For example, in the sexual process of multicellular organisms, gametogenesis can be interpreted as fibering, and gamete fusion can be interpreted as gluing. This modular universality of individual development in biological systems is also seemingly reflected in physical systems. Thus, sex-specific fibering in biology can be compared with the spin-specific differentiation in quantum physics systems.
For a local representation, biology models must reflect the dual nature of BOs, where they belong to two spaces simultaneously: to the sequence space and the observable space, in which the 'shape algebra' of biopolymers operates [5]. However, when modeling the processes responsible for mapping genotype information into phenotypic BO representations, the global properties of the biological (at least) world must be considered.

Recently, Baez and Stay [34] showed that the abstract Rosette stone of organization reveals the common operational units in category theory, physics, topology, logic, and computation. Our investigations with BO support including biology in this list.

\section{Acknowledgment}

The research was executed as a part of program of basic research of the Presidium of the Russian Academy of Sciences "Biosphere Origin and Evolution” (subprogram II) № 28 and the FEB RAS Program "Bioinformatics and Math. Biology" № 12-06-003.

\section{References}

[1] V. Kompanichenko, "Systemic approach to the origin of life", Front. Perspect., vol. 13, pp. 22-40, 2004.

[2] E. N. Trifonov, "Vocabulary of definitions of life suggests a definition", J. Biomol. Struct. Dyn., vol. 29, pp. 259-264, 2011.

[3] D. Raine and P. L. Luisi, "Open Questions on the Origin of Life (OQOL)”, Origins Life Evol. Biospheres, vol. 42, pp. 379-383, 2012.

[4] Yu. N. Zhuravlev and V. A. Avetisov, "The definition of life in the context of its origin", Biogeoscience, vol. 3, pp. 281-291, 2006.

[5] S. Ji and G. Ciobanu, "Conformon-driven biopolymer shape changes in cell modeling”, Biosystems, vol. 70, pp. 165-181, 2003.

[6] Yu. N. Zhuravlev and V. A. Avetisov, "Structure-function analysis of transformation events", In Genetic Transformation, M. Alvarez, Ed. InTech, 2011, pp. 29-52.

[7] Yu. N. Zhuravlev, "Definition by means of indefiniteness (comment)", J. Biomol. Struct. Dyn., vol. 29, pp. 643-644, 2012.

[8] C. H. Waddington, Principles of development and differentiation. New York: Macmillan Company, 1966.

[9] R. Rosen, Life Itself: A Comprehensive Inquiry into the Nature, Origin, and Fabrication of Life. Columbia: Columbia University Press, 1991.

[10] H. Pattee and J. Raczaszek-Leonardi, "Laws, language and life", Biosemiotics, vol. 7, pp. 3-333. 2013.

[11] B. Brogaard, "Species as individuals", Biology \& Philosophy, vol. 19, pp. 223-242, 2004.

[12] S. R. Gill, M. Pop, R. T. DeBoy, P. B. Eckburg, P. J. Turnbaugh, B. S. Samuel, J. I. Gordon, D. A. Relman, C. M. Fraser-Liggett, and K. E. Nelson, "Metagenomic analysis of the human distal gut microbiome", Science, vol. 312, pp. 1355-1359, 2006.

[13] J. Dupré and M. A. O'Malley, "The metagenomic world-view: a comment on Eric T. Juengst's 'Metagenomic metaphors", In New Visions of Nature, M. Drenthen et al. Eds. Springer Science+Business Media B.V. 2009, pp. 147-153.

[14] H. C. Fan, W. Gu, J. Wang, Y. J. Blumenfeld, Y. Y. El-Sayed, and S. R. Quake, "Non-invasive prenatal measurement of the fetal genome", Nature, vol. 487, pp. 320-324, 2012.

[15] S. Stegemann and R. Bock, "Exchange of genetic material between cells in plant tissue grafts", Science, vol. 324, pp. 649-651, 2009.

[16] cell 2014. Encyclopaedia Britannica Online. Retrieved 08 April, 2014, from http://www.britannica.com/ EBchecked/topic/101396/cell 
[17] P. J. Lewis, "Life in configuration space", Br. J. Philos. Sci., vol. 55, pp. 713-729, 2004.

[18] D. Michel, "Life is a self-organizing machine driven by the informational cycle of Brillouin", Origins Life Evol. Biospheres, vol. 43, pp. 137-150, 2013.

[19] S. Kauffman, R. K.Logan, R. Este, R. Goebel, D. Hobill, and I. Shmulevich, "Propagating organization: an enquiry", Biol. Philos., vol. 23, pp. 27-45, 2008.

[20] G.-F. Richard, A. Kerrest, and B. Dujon, "Comparative genomics and molecular dynamics of DNA repeats in eukaryotes", Microbiol. Mol. Biol. Rev., vol. 72, pp. 686-727, 2008.

[21] T. Nipkow, L. C. Paulson, and M. Wenzel, "Isabelle/HOL: a proof assistant for higher order logic. 6. Sets, functions, and relations", LNCS, vol. 2283, pp. 105-126, 2002.

[22] P. K. Lehre and P. C. Haddow, "Phenotypic complexity and local variations in neutral degree", Biosystems, vol. 87, pp. 233-242, 2007.

[23] D. Husemöller, Fibre bundles. 3rd ed. New York: Springer-Verlag, 1994.

[24] F. Crick, "Central dogma of molecular biology" Nature, vol. 227, pp. $561-563,1970$.

[25] E. Werner, "Genome semantics, in silico multicellular systems and the Central Dogma”, FEBS Lett., vol 579, pp. 1779-1782, 2005.

[26] A. F. Schier, "The maternal-zygotic transition: death and birth of RNAs", Science, vol. 316, pp. 406-407, 2007.
[27] J. S. Mattick, P. P. Amaral, M. E. Dinger, T. R. Mercer, and M. F. Mehler, "RNA regulation of epigenetic processes", BioEssays, vol. 31, pp. 51-59, 2009.

[28] A. A. Hoskins and M. J. Moore, "The spliceosome: a flexible, reversible macromolecular machine", Trends Biochem. Sci., vol. 37, pp. 179-188, 2012.

[29] H. R. Ueda, S. Hayashi, S. Matsuyama, T. Yomo, S. Hashimoto, S. A. Kay, J. B. Hogenesch, and M. Iino, "Universality and flexibility in gene expression from bacteria to human", Proc. Natl. Acad. Sci. U. S. A., vol. 101, pp. 3765-3769, 2004.

[30] P. O'Donoghue and Z. Luthey-Schulten, "On the evolution of structure in aminoacyl-tRNA synthetases", Microbiol. Mol. Biol. Rev., vol. 67, pp. 550-573, 2003.

[31] A. Marintchev and G. Wagner, "Translation initiation: structures, mechanisms and evolution”, Q. Rev. Biophys., vol. 37, pp. 197-284, 2004.

[32] S. Bentolila, "'Live memory' of the cell, the other hereditary memory of living systems", Biosystems, vol.80, pp. 251-261, 2005.

[33] D.. Galas, M. Nykter, G. W. Carter, N. D. Price, and I. Shmulevich, "Biological information as set-based complexity", IEEE Trans. Inf. Theory, vol. 56, pp. 667-677, 2010.

[34] J.C. Baez and M. Stay, "Physics, topology, logic and computation: a Rosetta stone", Lect. Notes Phys., vol. 813, pp. 95-172, 2011. 